\title{
Linking lipids and inflammasomes
}

The lipid bilayer of the cell membrane is involved in numerous cell functions, such as migration and cell signalling. Sterol regulatory element binding proteins (SREBPs) are key regulators of cellular lipid levels and, recently, these proteins have been shown to contribute to the repair of bacterial toxin-induced cell membrane damage in fibroblasts, downstream of caspase 1. Osborne and colleagues now show that the SREBP isoform SREBP1a links lipogenesis and inflammasome-mediated inflammatory responses in macrophages.

An analysis of SREBP isoform expression in various tissues and cells revealed that SREBP1a was most highly expressed by macrophages.
In addition, SREBP1a-deficient mice showed enhanced survival following toxic shock induced by high-dose lipopolysaccharide (LPS) administration or following caecal ligation and puncture (CLP)-induced sepsis compared with wild-type mice, suggesting that SREBP1a has a role in the innate immune response. Furthermore, serum interleukin-1 $\beta$ (IL-1 $\beta$ ) levels were reduced in both models.

The authors next compared global mRNA expression patterns in wild-type and SREBP1a-deficient macrophages and found that NOD-, LRR- and pyrin-domain containing 1A (Nlrp1a) and Nlrp1c mRNA levels were severely reduced in the

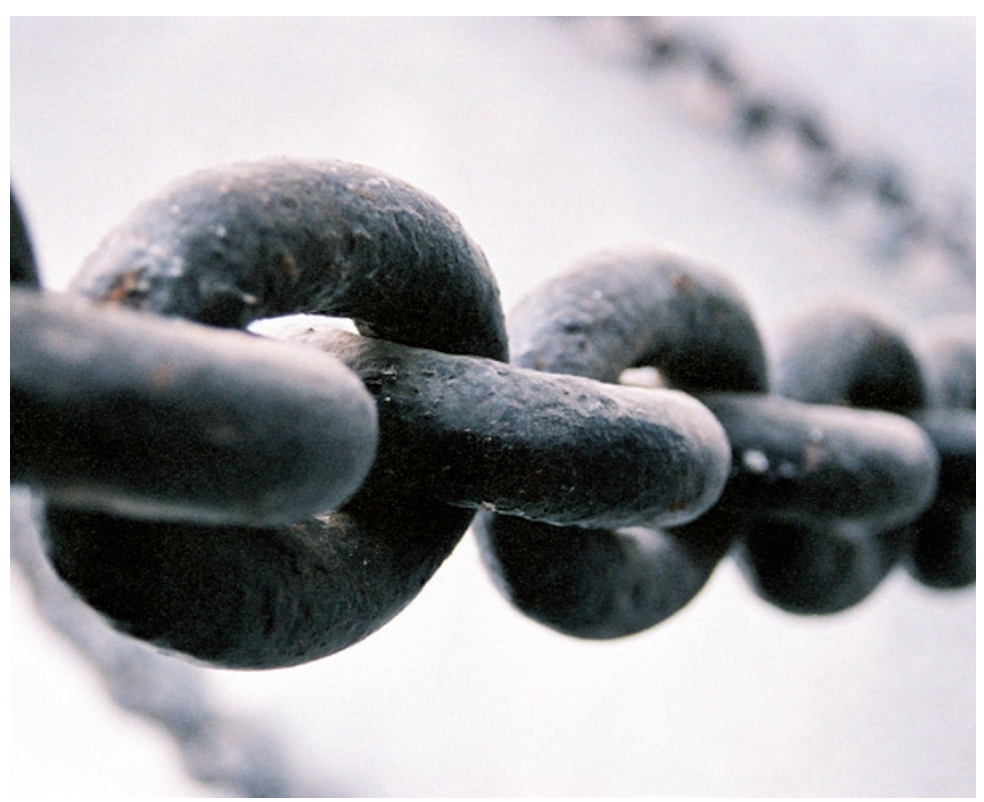

absence of SREBP1a. NLRP proteins can form multiprotein complexes (termed inflammasomes) that activate caspase 1 , which processes pro-IL-1 $\beta$ into the active cytokine. Indeed, SREBP1a-deficient macrophages had reduced caspase 1 activity in response to LPS stimulation and reduced LPS plus ATP-induced IL-1 $\beta$ secretion, suggesting a link between SREBP1a and inflammasome activation. Further analysis showed that re-expression of SREBP1a in deficient macrophages resulted in the return of Nlrpla but not Nlrp1c expression to that of wild-type levels. In addition, SREBP1a was shown to directly bind to and activate the Nlrpla promoter. So, Srebp1a regulates the NLRP1A inflammasome in macrophages.

Finally, SREBP1a expression was required for LPS-induced fatty acid synthesis in macrophages. Furthermore, LPS stimulation induced the recruitment of the transcription factors NF- $\kappa \mathrm{B}$ and SP1 to the Srebp1a promoter.

So, SREBP1a links LPS-induced lipogenesis with NLRP1A inflammasome activation in macrophages. This coupling of these two pathways highlights an important evolutionary role for lipid production in the response to pathogen challenge.

Olive Leavy

ORIGINAL RESEARCH PAPER Im, S.-S. et al. Linking lipid metabolism to the innate immune response in macrophages through sterol regulatory element binding protein-1a. Cell Metab. 13, 540-549 (2011) 